\title{
From swarm robotics to smart materials
}

\author{
Nikolaus Correll $\cdot$ Roderich Groß
}

Published online: 10 August 2010

(C) Springer-Verlag London Limited 2010

Swarm intelligence refers to the phenomenon of a system of spatially distributed individuals that coordinate their actions in a decentralized and self-organized manner, so as to exhibit complex collective behavior. Such systems tend to have large numbers of individual agents that interact with each other in simple ways. This allows swarm-intelligent systems to be inherently robust and flexible. As these principles are scale-free, systems with these properties can range in size from the nano to the macro scale.

Swarm-intelligent systems are common throughout nature. Examples are bacteria colonies, neural networks, social insects, and flocks/herds of vertebrates. In addition, humans have produced a variety of (artificial) swarm systems ranging from swarm-based optimization algorithms to sensor networks, swarms of robots, and smart materials. In each of these natural or artificial systems, populations of agents change their spatiotemporal configuration solely based on the agents' local interactions with each other and the environment.

This special issue on "Swarm Robotics" provides an overview of recent results and trends in this emerging field. Contributions to this special issue range from programming paradigms for swarming systems to specific distributed algorithms and modular robotic systems. The unifying

\footnotetext{
N. Correll $(\bowtie)$

Department of Computer Science, University of Colorado at Boulder, 430 UCB, Boulder, CO 80309, USA

e-mail: nikolaus.correll@colorado.edu

R. Groß

Department of Automatic Control and Systems Engineering,

The University of Sheffield, Mappin Street,

Sheffield S1 3JD, UK

e-mail: r.gross@sheffield.ac.uk
}

theme of these works is individual simplicity: complex global behavior emerges from purely local interactions and simple local rules. Examples covered in this special issue range from spatial behaviors such as flocking and dispersion, computational behaviors such as shortest-path routing and collective decisions, up to full-body behaviors of modular robot ensembles.

In their paper, "Composable continuous-space programs for robotic swarms", Bachrach, Beal, and McLurkin present the functional programming language Proto that allows individual behavior to be described by expressions over a global field. By computing the global field not only from local measurements but also based on data received from other swarm members within the local neighborhood, previous state, and control logic, Proto allows complex swarming behaviors to be composed with highly compact code. Proto code is then compiled into op-codes for the Proto Virtual Machine, which needs to provide abstractions for sensing, actuation, estimation of the geometric relations between neighboring swarm members, and local communication. Algorithms such as shortest-path routing are demonstrated on a swarm of 40 miniature mobile robots, as well as in computer simulations.

In their paper, "Collective decision-making based on social odometry", Gutiérrez, Campo, Monasterio-Huelin, Magdalena, and Dorigo investigate a novel collective decision-making mechanism using a colony of mobile robots that accomplish a foraging task. The robots are required to establish a path from a central place to the closest of multiple resource sites. To reach a consensus, they make use of social odometry. The latter mechanism enables the robots to estimate the position of resource sites by exchanging and aggregating odometry-based positional information and confidence levels. The collective decisionmaking mechanism is successfully validated by experiment 
using ten robots of the e-puck platform, as well as by computer simulations.

In "Steering self-organized robot flocks through externally guided individuals", Çelikkanat and Şahin study the impact of swarm members with biased directions on the cohesion and stability of flocking motion. Inspired by similar observations of flocking in animals such as schools of fish and flocks of birds, the authors systematically study the impact of parameters such as the ratio of informed individuals and the number of individuals in the flock. They validate predictions previously made by biologists in experiments with seven mobile robots, as well as by computer simulations.

In "Anatomy-based organization of morphology and control in self-reconfigurable modular robots", Christensen, Campbell, and Stoy address design strategies for robotic systems that are composed of large numbers of autonomous physically interconnected robotic modules. The authors present an approach that is inspired by the anatomical structures found in complex multicellular animals such as bones and muscles. The anatomy-inspired parts are made of modules and then combined to form complete functional bodies of reconfigurable modular robots. The authors argue that this approach is particular relevant for systems of micron-sized modules. Using a computer simulation of the Catom model, they show how global behaviors such as gaits can be defined by controlling the intermediate level parts. Moreover, they critically assess the abilities and limitations of the ATRON modular robotic platform by demonstrating several examples of anatomy-inspired parts.

This special issue of the journal "Neural Computing and Applications" has received 19 submissions, of which four submissions were accepted after several rounds of review. For the selection of papers, emphasis was placed on swarm robotic systems that achieve interesting global behavior with simplistic individual control, independent of its implementation. Synthesizing artificial neural networks that are equivalent to the controllers proposed in the papers of this special issue, especially for those that are bioinspired such as the distributed algorithm for informed flocking, might lead to important insight into the nervous systems of the animals that exhibit similar behavior. At the same time, hardware-based implementations of neural network or other minimalistic controllers, e.g. using polymer electronics, could help in the transition to smart materials-exemplified by the modular robotics contribution in this special issue-as it would further reduce the number of monolithic, atomic building blocks in the system. 